\title{
Bioactive metabolites of Cunninghamella, Biodiversity to Biotechnology
}

\author{
Waill A. Elkhateeb ${ }^{1 *}$, Abdu Ghalib AL Kolaibe ${ }^{2}$, Ghoson M. Daba ${ }^{1}$ \\ ${ }^{1}$ Chemistry of Natural and Microbial Products Department, Pharmaceutical Industries Division, National Research Centre, Dokki, Giza, 12622, Egypt.
}

*Corresponding Author: Waill A. Elkhateeb, Chemistry of Natural and Microbial Products Department, Pharmaceutical Industries Division, National Research Centre, Dokki, Giza, 12622, Egypt

Received date: March 28, 2021; Accepted date: April 19, 2021; Published date: April 24, 2021

Citation: Waill A. Elkhateeb, Abdu G. AL Kolaibe, Ghoson M. Daba (2021) Bioactive metabolites of Cunninghamella, Biodiversity to Biotechnology J. Pharmaceutics and Pharmacology Research, 4(3); DOI:10.31579/2693-7247/036

Copyright: (C) 2021, Waill A. Elkhateeb, This is an open access article distributed under the Creative Commons Attribution License, which permits unrestricted use, distribution, and reproduction in any medium, provided the original work is properly cited.

\begin{abstract}
Cunninghamella is a fungal genus that belongs to family Cunninghamellaceae and has been involved as promising tool in many important mycotechnological applications. Cunninghamella is an endophytic fungus, their secondary metabolites are of potential biological activities especially as antimicrobial agents. The aim of this review is to highlight the description, ecology, and important in medicinal and industrial applications of the genus Cunninghamella in general. Moreover, describing the importance and potentials of this fungus in order to encourage for further studies to search, isolate, and purify already known metabolites. Also, screen for, and discover novel metabolites produced by this potent fungi in order to be involved in additional applications.
\end{abstract}

Keyword: cunninghamella; mucorales; bioactive metabolites; biotransformation

\section{Introduction}

Fungi play important role in human life such as in agriculture, food industry, medicine, textiles, bioremediation, natural cycling, as biofertilizer and in many other ways. Fungi are ubiquitous on earth and represent essential components of many ecosystems where they are involved in many vital processes [1]. Mucorales is the largest order of fungi, it is classified into the subphylum named Mucormycotina [2], that is formed of about 57 genera with 15 families and that comprise about 334 species [3]. Mucorales members are found in soil, water, dung, plants, stored grains, and fungal masses [4, 5]. Many of mucorales members show rapid growth and they colonize and sporulate on a variety of carbohydrate-rich, and terrestrial substrates.

The genus Cunninghamella (Cunninghamellaceae, Mucorales) was established by Matruchot in 1903. The species of this genus are characterized by the formation of the uni-spored sporangia and pedicellate on the vesicle surface [6]. About 14 species are identified in this genus [7, 8]. These species are usually found in soil, organic substrates, and stored grains, etc. $[9,10]$. Some species are reported to cause infection such as human mucormycosis $[11,12]$, however other species are reported to be very helpful in treating many diseases as well as that can destroy tumors [13]. Based on the morphological characterization, the taxonomy of Cunninghamella has been identified. This include the colony color, shape and size of vesicles, branching pattern of sporophore, sporangiola shape, and the length of the spines $[6,14]$.
Another study reported that Cunninghamella genus was monographed upon the maximum growth temperatures together with the morphological characteristics [6]. Moreover, other studies have sequenced the internal transcribed spacer (ITS), 28S, and elongation factor $1 \alpha$ gene regions of the accepted taxa and represented the phylogeny to be compatible with Zeng and Chen's taxonomical results $[15,16]$.

The endophytic fungus, Cunninghamella sp., is considered an enormous source of chemical leads with promising biological activities. Different Cunninghamella species have proved their ability to produce plenty of secondary metabolites including fatty acids (Palmitic acid, oleic acid and stearic acid) in addition to two steroidal compounds; $\alpha$-amyrin (A4), and $\beta$-sitosterol with various biological activities, such as antifungal and antibacterial [17-19]. Cunninghamella species capable to produced different enzymes that can perform both phase I and phase II biotransformations [20].

Secondary metabolites differ from primary nutrients in that they are not essential for growth, but they play a vital role in the survival and adaptation of producing organisms in nature [21]. The genus Cunninghamella contains species of importance in medical mycology and in biotechnological processes. Cunninghamella is a filamentous fungus found in soil and plant material, particularly at Mediterranean and subtropical zones. Cunninghamella bertholletiae, Cunninghamella elegans and Cunninghamella echinulata are the most common species. Cunninghamella colonies are rapidly growing, full mature in 4 days, 
colony shape is cottony and white to tannish-grey in color. Cunninghamella elegans produces purely gray colonies. [22, 23].

\section{The genus Cunninghamella description and ecology}

Colonies fast growing, completely filling the plate on Czapek's, malte extract, or potato dextrose agar, after 3-5 days of incubation at $25^{\circ} \mathrm{C}$, floccose, up to $3 \mathrm{~cm}$ high, white to dark grey; mycelium non septate, often becoming septate without order in age; hyphae broad up to thick. Sporangiophores (Conidiophores) erect, branched in verticillate or irregular, each branch terminating in globose, obovoid pyriform to clavate vesicle furnished with small sporangioles (Conidia) bearing denticles, usually sporangiophores swollen at point of attachment of lateral branches; sporangioles arising from denticles synchronously (Figure, 1, 2). 1-Spored globose, ovoid or elliptical, smooth verrucose or echinulate, hyaline mostly heterothallic. Zygospores globose to somewhat flattened, dark brown, tuberculate, suspensors equal. When mature colonies greyish white; terminal vesicles up to $40 \mathrm{um}$ in diameter, sporangioles smooth, species is Cunninghamella elegans. When mature colonies pale grey, terminal vesicles up to 50 um in diameter, sporangioles echinulate, species is Cunninghamella echinulata. Cunninghamella echinulata most common and is worldwide distribution [24-26].
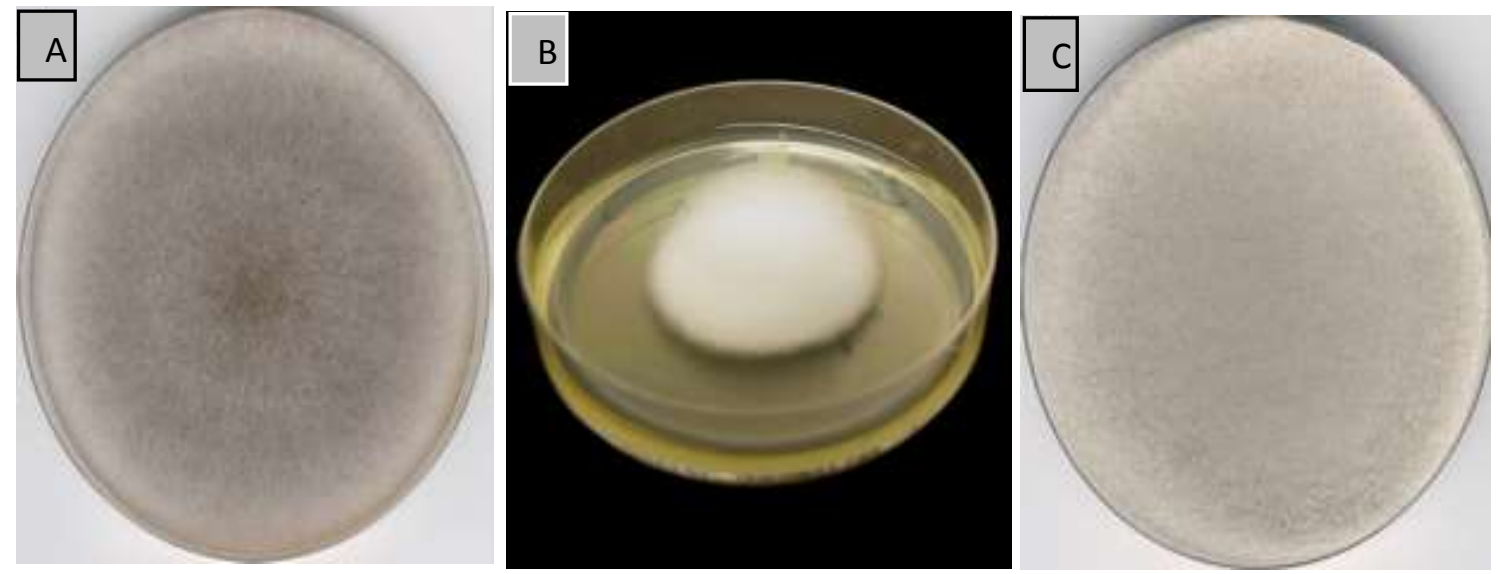

Figure 1. Cunninghamella different species with different shapes on different media (cited in: $\underline{w w w . t h u n d e r h o u s e 4-y u r i . b l o g s p o t . c o m}$ and https://www.natur.cuni.cz).

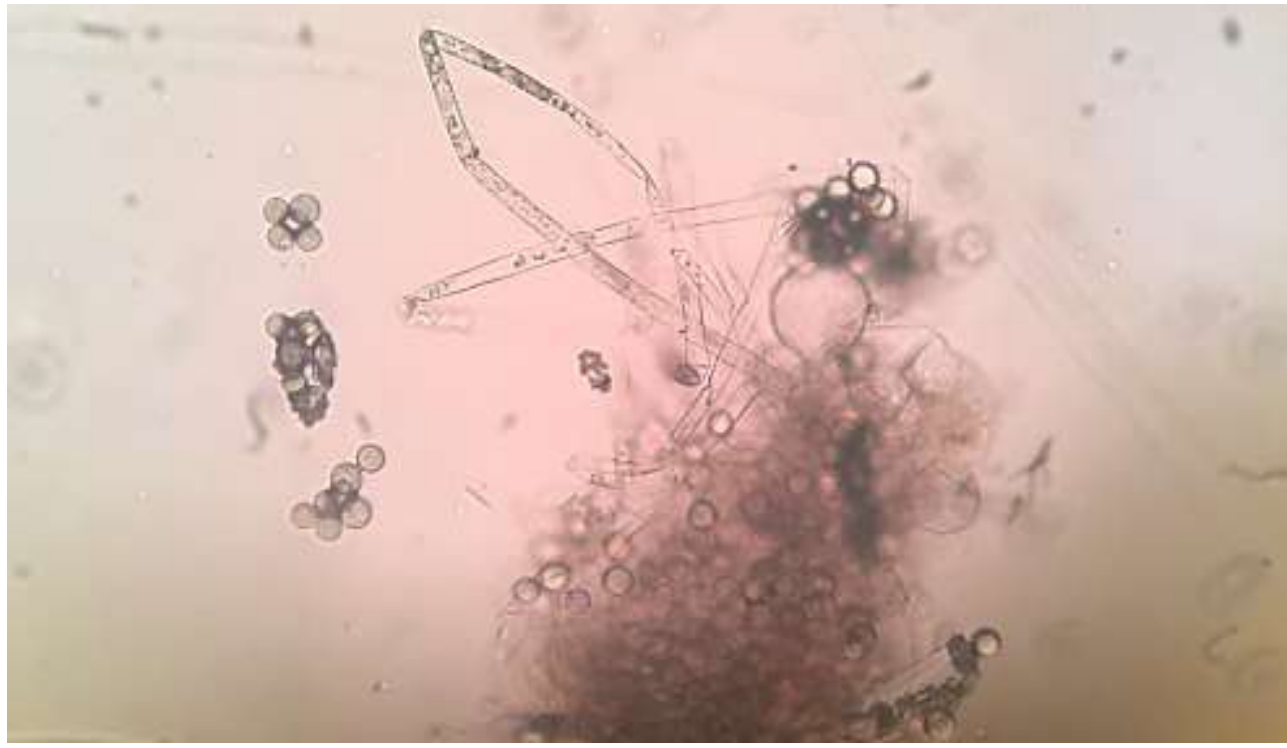

Figure 2. Cunninghamella elegans with smooth globose conidia isolated and identified by Dr. Waill Elkhateeb (Photographs was taken by Dr. Waill A. Elkhateeb, Locality: National Research Center of Egypt).

\section{Cunninghamella secondary metabolites and their biological activities}

Various fungi produce many secondary metabolites that have diverse molecular structure [27]. Several phytochemical groups have been isolated from fungi, these include alkaloids, carbohydrates, fatty acid, phenolic compound, polyketides, and terpenes [28]. Nowadays, over 90,000 species of fungi have been identified [29], nevertheless, not all these fungi have been fully studied for their potential biological and chemical properties [30, 31]. Cunninghamella is a fungal genus that belongs to family Cunninghamellaceae. This family may exist in soil, plant and animal material, and Brazil nuts, etc. [32]. Members of this 
genus are characterized by possessing cytochrome P-450 monooxygenase systems similar to those found in mammals [33]. Thus, they have been applied to many drugs where they act in a manner that similar to those of mammalian enzyme systems [34]. For this reason, Cunninghamella species have been applied as biotransformation media, because of its capacity to convert different molecules to valuable medicinal substances [35].

Among the objectives of the recent European Union legislation governing the testing and evaluation of chemicals, REACH (Regulation, Evaluation, Authorisation and Restriction of Chemicals), is to minimize depending on animals in testing experiments. Since some microorganisms including the actinomycetes bacteria and Cunninghamella fungi are able to metabolize different xenobiotic compounds in a way which is analogous to that of mammals [22, 23], [36, 37]. These microorganisms have been considered as potent models of mammalian metabolism [38], even though many concerns are found about their predictive value (8). However, some microorganisms are very crucial in the generation of useful quantities of drug metabolic intermediates [39].

Various Cunninghamella species have shown different biological activities. For example, Cunninghamella echinulata has been reported to biotransform the drug "loratadine" to its active metabolite "desloratadine" and which was proved to be more potent (about four times that of loratadine) when consumed at low doses. Desloratadine is a potent antihistaminic drug that has showed its efficacy in allergic disease [40, 41]. Also, Cunninghamella elegans ATCC 9245, showed its ability to biotransform doxepin which is a tricyclic antidepressant drug [42]. Interestingly, previous studies revealed diverse pathways for Cunninghamella blakesleana AS 3.153 to metabolize verapamil, in a way similar to humans that is why this species could be a useful model for studying mammalian drug metabolism system [43].

This species also showed its importance in treatment of cancer. Some studies reported that the potential of employing Cunninghamella species to transform many xenobiotic compounds in an analogous way similar to mammals. Cunninghamella species were able to biotransform the anticancer drug flutamide into four fluorometabolites. Among the produced metabolites, the most predominant one was the 2-hydroxyflutamide which is known to be a major pharmacologically active compound in mammals. Cunninghamella species also play an important role in inflammation treatment. They can biotransform flurbiprofen "the fluorinated anti-inflammatory drug”, where, mono- and dihydroxylated metabolites were detected by gas chromatography-mass spectrometry (GC-MS) and fluorine-19 nuclear magnetic resonance spectroscopy ( ${ }^{19}$ FNMR). 4'-hydroxyflurbiprofen was proved to be the major metabolite being converted by both microbial and mammalian systems [44].

The antimicrobial activities of Cunninghamella blakesleeana ethanol extract has been examined against different Gram-positive and Gramnegative bacteria, as well as other fungi. Some of the isolated compounds showed their ability to inhibit the growth of some microorganisms. The maximum antimicrobial activity of Cunninghamella blakesleeana alcohol extract was reported against Staphylococcus aureus this was followed by Streptococcus pyogenes. On the other side, the maximum antimicrobial activity was reported against for both Candida albicans and Pseudomonas aeruginosa [17]. Cunninghamella blakesleeana has been also reported to produce many fatty acids. About ten of them were detected. Stearic acid was the major fatty acid isolated from C. blakesleeana (74.61\%), palmitic acid was considered the second high percentage (10.35\%), however, docosanoic acid showed the least percentage $(0.01 \%)$ [17]. These lipids can be applied in various industries such as essential oils, natural food colors, spice, and oleoresins. Also they can be used in cosmetics and medicinal uses [45]. Additionally, Cunninghamella elegans showed its capacity to produce polysaccharides when the fungus was grown on corn steep liquor and cassava wastewater. The obtained chitin and chitosan showed deacetylation degrees of 40.98 and $88.24 \%$, and a crystalline index of $35.80 \%$ and $23.82 \%$, respectively. The produced chitin and chitosan are found to be non-irritating since they do not promote vascular change such as vasoconstriction, haemorrhage, and coagulation [46].

Recently, more effort has been directed towards the production of fungal chitin and chitosan as an alternative source of biopolymers in order to overcome the undesirable side effects of the traditional extraction methods of these polysaccharides [47, 48]. Additionally, the microbial chitin and chitosan have more consistent quality and homogenous characteristics $[49,50]$. Chitin and chitosan extracted from mycelial biomass of $C$. elegans were able to remove various metals such as copper, lead, and iron from their aqueous solutions, where chitosan $(1 \% \mathrm{w} / \mathrm{v})$ showed the highest capacity for copper biosorption (75\%). While chitin $(1 \% \mathrm{w} / \mathrm{v})$ showed the maximum capacity to remove iron metal $(56 \%)$, both experiment were conducted at $\mathrm{pH} 4$ and $2.0 \mathrm{mM}$ metals concentration [51].

Moreover, the dye biosorption potential of has been proven to be very effective, where it was found to remove some industrial dyes such as R80 and B214. Dye and toxic metals biosorption with fungal biomasses are considered important alternatives to other traditional wastewater treatment techniques that aim to reuse water. Fungal biomasses have the advantages of being cheaper, easier in operation and insensitive to toxic pollutants [52]. Also, Cunninghamella elegans showed its ability to remove toxic metals, where it was able to grow in the presence of large amount of toxic copper [53]. Cunninghamella blakesleeana, Cunninghamella homothallica, and Cunninghamella elegans to show their ability to grow in the presence of different metals such as cadmium, cobalt, and chromium [54]. These results indicate that Cunninghamella species can offer a new natural way for bioremediation of toxic industrial wastes such as dyes as well as other toxic metals.

\section{Conclusion}

The application of microbial models has gained a great attention as a complementary tool to study drugs metabolism. Microorganisms may be employed as an efficient and valuable alternative to animals or even synthetic chemistry to study many drug derivatives. The expression of the cytochromes $\mathrm{P} 450$ in heterologous systems (such as fungi) has been successfully employed as a tool for investigating the metabolism of a wide variety of drugs. Among these fungi species is Cunninghamella species that can find a great application as a reliable alternative to in vitro models to study various drugs metabolism. Cunninghamella species have been reported to produce some metabolites such as polysaccharides and fatty acids, that show valuable applications. Moreover, Cunninghamella species can be employed as a powerful bioremediation tool to get rid of many harmful industrial dyes as well as toxic metals. 


\section{References}

1. Dighton J, White JF. (2017). The fungal community: its organization and role in the ecosystem. CRC press.

2. Hibbett DS, Binder M, Bischoff JF, Blackwell M, Cannon PF, Eriksson OE, Huhndorf S, James T, Kirk PM, Lücking R. (2007). A higherlevel phylogenetic classification of the Fungi. Mycological research 111: 509-547.

3. McLaughlin D, McLaughlin E, Lemke P. (2001). The Mycota. Vol. VII. Part A. Systematics and Evolution. Springer-Verlag, Berlin.

4. Ariyawansa HA, Hyde KD, Jayasiri SC, Buyck B, Chethana KT, Dai DQ, Dai YC, Daranagama DA, Jayawardena RS, Lücking R. (2015). Fungal diversity notes 111-252-taxonomic and phylogenetic contributions to fungal taxa. Fungal diversity 75: 27-274.

5. Benny GL. (2008). Methods used by Dr. RK Benjamin, and other mycologists, to isolate zygomycetes. Aliso: A Journal of Systematic and Evolutionary Botany 26: 37-61.

6. Zheng R, Chen G. (2001). A monograph of Cunninghamella. Mycotaxon 80: 1-75.

7. Alves A, de Souza C, de Oliveira R, Cordeiro T, de Santiago A. (2017). Cunninghamella clavata from Brazil: a new record for the western hemisphere. Mycotaxon 132: 381-389.

8. Guo J, Wang H, Liu D, Zhang J-N, Zhao Y-H, Liu T-X, Xin Z-H. (2015). Isolation of Cunninghamella bigelovii sp. nov. CGMCC 8094 as a new endophytic oleaginous fungus from Salicornia bigelovii. Mycological Progress 14: 1-8.

9. Baijal U, Mehrotra B. (1980). The genus Cunninghamella-a reassessment. Sydowia 33, 1-13.

10. Liu C-W, Liou G-Y, Chien C-Y. (1993). New records of the genus Cunninghamella (Mucorales) in Taiwan. Notes.

11. Gomes MZ, Lewis RE, Kontoyiannis DP. (2011). Mucormycosis caused by unusual mucormycetes, non-Rhizopus,-Mucor, andLichtheimia species. Clinical Microbiology Reviews 24: 411-445.

12. Kwon-Chung KJ. (2012). Taxonomy of fungi causing mucormycosis and entomophthoramycosis (zygomycosis) and nomenclature of the disease: molecular mycologic perspectives. Clinical Infectious Diseases 54: S8-S15.

13. Betts RE, Walters DE, Rosazza JP. (1974). Microbial transformations of antitumor compounds. 1. Conversion of acronycine to 9hydroxyacronycine by Cunninghamella echinulata. Journal of medicinal chemistry 17: 599-602.

14. Weitzman I, Crist MY. (1980). Studies with clinical isolates of Cunninghamella II. Physiological and morphological studies. Mycologia 72: 661-669.

15. Liu X, Huang H, Zheng R. (2003). Relationships within Cunninghamella based on sequence analysis of its rDNA (vol 80, pg 77, 2001). Mycotaxon 87: 541.

16. Walther G, Pawłowska J, Alastruey-Izquierdo A, Wrzosek M, Rodriguez-Tudela J, Dolatabadi S, Chakrabarti A, De Hoog G. (2013). DNA barcoding in Mucorales: an inventory of biodiversity. Persoonia: Molecular Phylogeny and Evolution of Fungi 30: 11.

17. Alasmary FA, Awaad AS, Alqahtani SM, El-Meligy RM, Abdullah DA, Alqasoumi SI. (2020). Evaluation of the chemical constituents and potential biological activities of Cunninghamella blakesleeana. Saudi Pharmaceutical Journal 28: 1197-1202.
18. Cha C-J, Doerge DR, Cerniglia CE. (2001). Biotransformation of Malachite Green by the Fungus Cunninghamella elegans. Applied and environmental microbiology 67: 4358-4360.

19. Verma VC, Gange AC. (2013). Advances in endophytic research. Springer.

20. Zhang D, Yang Y, Leakey JE, Cerniglia CE. (1996). Phase I and phase II enzymes produced by Cunninghamella elegans for the metabolism of xenobiotics. FEMS microbiology letters 138: 221-226.

21. Soltani J. (2016). Secondary metabolite diversity of the genus Aspergillus: recent advances. New and future developments in microbial biotechnology and bioengineering, 275-292.

22. Asha S, Vidyavathi M. (2009). Cunninghamella-a microbial model for drug metabolism studies-a review. Biotechnology advances 27: 16-29.

23. Sun L, Huang H-H, Liu L, Zhong D-F. (2004). Transformation of verapamil by Cunninghamella blakesleeana. Applied and environmental microbiology 70: 2722-2727.

24. Elkhateeb WA. (2005). Some mycological, phytopathological and physiological studies on mycobiota of selected newly reclaimed soils in Assiut Governorate, Egypt (M. Sc. Thesis, Faculty of Science, Assuit University, Egypt. 2005; p 238.

25. Elkhateeb WA, Daba GM. (2018). Where to Find? A Report for Some Terrestrial Fungal Isolates, and Selected Applications Using Fungal Secondary Metabolites. Biomed Journal Science \&Technology Research 4(4): 1-4.

26. Elkhateeb WA, Zohri AA, Mazen M, Hashem M, Daba GM. (2016). Investigation of diversity of endophytic, phylloplane and phyllosphere mycobiota isolated from different cultivated plants in new reclaimed soil, Upper Egypt with potential biological applications, Inter J MediPharm Res, 2(1): 23-31.

27. Gao H, Li G, Lou H-X. (2018). Structural diversity and biological activities of novel secondary metabolites from endophytes. Molecules 23: 646 .

28. Metsämuuronen S, Sirén H. (2019). Bioactive phenolic compounds, metabolism and properties: A review on valuable chemical compounds in Scots pine and Norway spruce. Phytochemistry Reviews 18: 623-664.

29. Salar RK. (2018). Thermophilic fungi: basic concepts and biotechnological applications. CRC Press.

30. Amare MG, Keller NP. (2014). Molecular mechanisms of Aspergillus flavus secondary metabolism and development. Fungal Genetics and Biology 66: 11-18.

31. Pusztahelyi T, Holb IJ, Pócsi I. (2015). Secondary metabolites in fungus-plant interactions. Frontiers in plant science 6: 573.

32. Nguyen TT, Choi Y-J, Lee HB. (2017). Isolation and characterization of three unrecorded Zygomycete fungi in Korea: Cunninghamella bertholletiae, Cunninghamella echinulata, and Cunninghamella elegans. Mycobiology 45: 318-326.

33. Chen W, Lee M-K, Jefcoate C, Kim S-C, Chen F, Yu J-H. (2014). Fungal cytochrome p450 monooxygenases: their distribution, structure, functions, family expansion, and evolutionary origin. Genome biology and evolution 6: 1620-1634.

34. Kelly SL, Kelly DE. (2013). Microbial cytochromes P450: biodiversity and biotechnology. Where do cytochromes P450 come from, what do they do and what can they do for us? Philosophical 
Transactions of the Royal Society B: Biological Sciences 368: 20120476.

35. Palmer-Brown W, Miranda-CasoLuengo R, Wolfe KH, Byrne KP, Murphy CD. (2019). The CYPome of the model xenobioticbiotransforming fungus Cunninghamella elegans. Scientific reports 9: 1-9.

36. Cannell R, Knaggs AR, Dawson MJ, Manchee GR, Eddershaw PJ, Waterhouse I, Sutherland DR, Bowers GD, Sidebottom PJ. (1995). Microbial biotransformation of the angiotensin II antagonist GR117289 by Streptomyces rimosus to identify a mammalian metabolite. Drug metabolism and disposition 23: 724-729.

37. Moody JD, Freeman JP, Cerniglia CE. (1999). Biotransformation of doxepin by Cunninghamella elegans. Drug metabolism and disposition 27: 1157-1164.

38. Smith RV, Rosazza JP. (1974). Microbial models of mammalian metabolism. Aromatic hydroxylation. Archives of biochemistry and biophysics 161: 551-558.

39. Osorio-Lozada A, Surapaneni S, Skiles GL, Subramanian R. (2008). Biosynthesis of drug metabolites using microbes in hollow fiber cartridge reactors: case study of diclofenac metabolism by Actinoplanes species. Drug Metabolism and Disposition 36: 234-240.

40. Murdoch D, Goa KL, Keam SJ. (2003). Desloratadine. Drugs 63, 2051-2077.

41. Keerthana M, Vidyavathi M. (2018). Screening and evaluation of fungal resources for loratadine metabolites. Journal of biosciences 43: 823-833.

42. Moody JD, Zhang D, Heinze TM, Cerniglia CE. (2000). Transformation of Amoxapine by Cunninghamella elegans. Applied and environmental microbiology 66: 3646-3649.

43. Liu L, Huang H-H, Sun L, Zhong D-F. (2002). Capability of Cunninghamella blakesleana to transform verapamil. Chinese J Pharmacol Toxicol. 16: 292-298.

44. Amadio J, Gordon K, Murphy CD. (2010). Biotransformation of flurbiprofen by Cunninghamella species. Applied and environmental microbiology 76: 6299-6303.
45. Yadav P. (2006). Lipid biotechnology. Discovery Publishing House. 46. Berger LR, Stamford TC, Stamford-Arnaud TM, de Oliveira Franco L, Do Nascimento AE, Cavalcante HM, Macedo RO, Campos-Takaki D, Maria G. (2014). Effect of corn steep liquor (CSL) and cassava wastewater $(\mathrm{CW})$ on chitin and chitosan production by Cunninghamella elegans and their physicochemical characteristics and cytotoxicity. Molecules 19: 2771-2792.

47. Arbia W, Adour L, Amrane A, Lounici H. (2013). Optimization of medium composition for enhanced chitin extraction from Parapenaeus longirostris by Lactobacillus helveticus using response surface methodology. Food Hydrocolloids 31: 392-403.

48. Tajdini F, Amini MA, Nafissi-Varcheh N, Faramarzi MA. (2010). Production, physiochemical and antimicrobial properties of fungal chitosan from Rhizomucor miehei and Mucor racemosus. International journal of biological macromolecules 47: 180-183.

49. Bueter CL, Specht CA, Levitz SM. (2013). Innate sensing of chitin and chitosan. PLoS Pathog 9: e1003080.

50. Nwe N, Stevens WF. (2004). Effect of urea on fungal chitosan production in solid substrate fermentation. Process Biochemistry 39: 1639-1642.

51. Franco LdO, Maia Rd, Porto AL, Messias AS, Fukushima K, CamposTakaki GM. (2004). Heavy metal biosorption by chitin and chitosan isolated from Cunninghamella elegans (IFM 46109). Brazilian Journal of Microbiology 35: 243-247.

52. Prigione V, Tigini V, Pezzella C, Anastasi A, Sannia G, Varese G. (2008). Decolourisation and detoxification of textile effluents by fungal biosorption. Water Research, 42(12): 2911-2920.

53. Souza PM, Marinho PH, Lima MA, Nascimento AE, Takaki G. (2005). Copper influence on polyphosphate metabolism of Cunninghamella elegans. Brazilian Journal of Microbiology, 36(4): 315-320.

54. Mattar EH, Hammad LF, Zain ME. (2011). Effect of cadmium, cobalt, and chromium on growth of three Cunninghamella species. Journal of Saudi Chemical Society 15(4): 357-359.
This work is licensed under Creative Commons Attribution 4.0 License

To Submit Your Article Click Here: Submit Manuscript

DOI:10.31579/2693-7247/036
Ready to submit your research? Choose Auctores and benefit from:

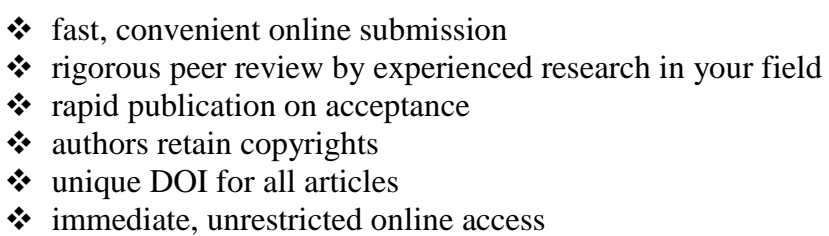

At Auctores, research is always in progress.

Learn more www.auctoresonline.org/journals/pharmaceutics-andpharmacology-research 